\title{
Short-Term Effects of Glucosyl Hesperidin and Hesperetin on Blood Pressure and Vascular Endothelial Function in Spontaneously Hypertensive Rats
}

\author{
Masaki Yamamoto, Atsushi SuZUKI* and Tadashi HaSE \\ Biological Science Laboratories, Kao Corporation, 2606 Akabane, Ichikai-machi, Tochigi 321-3497, Japan
}

(Received June 5, 2007)

\begin{abstract}
Summary Glucosyl hesperidin (G-hesperidin) is a water-soluble derivative of hesperidin. In the present study, the short-term effects of G-hesperidin and hesperetin, a putative metabolite of G-hesperidin, in spontaneously hypertensive rats (SHR) and in normotensive WistarKyoto rats (WKY) were investigated. Single oral administration of G-hesperidin (10 to $50 \mathrm{mg} / \mathrm{kg}$ ) induced a dose-dependent reduction in systolic blood pressure (SBP) in SHR, but had no effects in WKY. Intraperitoneal injection of hesperetin $(50 \mathrm{mg} / \mathrm{kg})$ into SHR also caused a significant reduction in SBP. The depressor effect was significantly inhibited by a nitric oxide synthase inhibitor, $N^{\mathrm{G}}$-nitro-L-arginine methyl ester. Moreover, hesperetin $\left(10^{-5} \mathrm{M}\right)$ enhanced endothelium-dependent relaxation induced by acetylcholine, but had no effect on endothelium-independent relaxation induced by sodium nitroprusside in isolated aortas from SHR. These data suggest that the hypotensive effect of hesperetin in SHR is associated with nitric oxide-mediated vasodilation. Therefore, this effect may be involved in the mechanisms by which G-hesperidin lowers blood pressure in hypertension.
\end{abstract}

Key Words glucosyl hesperidin, hesperetin, hypertension, endothelial function, nitric oxide

Epidemiological studies indicate that ingestion of fruits and vegetables inhibits the development of cardiovascular disease (1). Hesperidin is a well-known flavonoid, abundant in citrus fruit peel, which exhibits various physiological activities, such as reduction in capillary fragility (2), antioxidative effects (3), antihypertensive effects (4), and cholesterol-lowering actions (5). Glucosyl hesperidin (G-hesperidin) is a water-soluble derivative of hesperidin (6). Because G-hesperidin has the same metabolic profile as hesperidin (7), it is expected to have similar physiological activities. In fact, G-hesperidin has been reported to have an antihypertensive effect $(8)$ and to improve lipid metabolism (9, 10). However, the underlying mechanisms involved in the antihypertensive effects of G-hesperidin have not been examined in detail.

Therefore, the aim of the present study was to investigate the mechanisms for the hypotensive effect of Ghesperidin in spontaneously hypertensive rats (SHR). Ghesperidin is assumed to metabolize to hesperetin in the small intestine where it is absorbed (7). Thus, the effects of hesperetin on blood pressure in SHR and on vascular reactivity in isolated aortas from SHR and normotensive Wistar-Kyoto rats (WKY) were examined.

\section{Materials and Methods}

Animals. Male SHR (SHR/Izm) and WKY (WKY/

*To whom correspondence should be addressed.

E-mail: suzuki.atsushi2@kao.co.jp
Izm) rats were purchased from SLC, Inc. (Shizuoka, Japan). All animal experiments were approved by the Ethics Review Committee for Animal Experimentation of Kao Corporation.

Single oral administration of G-hesperidin. G-hesperidin (Lot. SP0917500, including 77\% mono-glucosyl hesperidin, Toyo Sugar Refining Co. Ltd., Tokyo, Japan) was orally administered at 10,30 , and $50 \mathrm{mg} / \mathrm{kg}$ to SHR or at $50 \mathrm{mg} / \mathrm{kg}$ to WKY $(16-20 \mathrm{wk}$ old, $n=10-$ 12). Systolic blood pressure (SBP) and heart rate were measured $0,3,6,9,12$, and $24 \mathrm{~h}$ after administration of G-hesperidin by the tail-cuff method using an automatic blood pressure monitoring system (BP-98A, Softron, Tokyo, Japan). Urine was collected for $12 \mathrm{~h}$ after a single oral administration of G-hesperidin $(50 \mathrm{mg} / \mathrm{kg})$ or physiologic saline (control group) in metabolic cages $(n=8)$. Urinary sodium concentration was measured by Mitsubishi Chemical Medience Corp. (Tokyo, Japan).

Hypotensive effects of hesperetin in SHR. Hesperetin (Sigma, St. Louis, MO) was suspended in 0.5\% methylcellulose and intraperitoneally injected into SHR at $50 \mathrm{mg} / \mathrm{kg}$. SBP was measured $1 \mathrm{~h}$ after hesperetin administration. Enalapril, an angiotensin-converting enzyme (ACE) inhibitor, was orally administered at a dose of $20 \mathrm{mg} / \mathrm{kg} 4 \mathrm{~h}$ before administration of hesperetin. Nicardipine, a calcium channel blocker, or prazosin, an adrenergic $\alpha$ receptor antagonist, were orally administered with hesperetin at doses of 5 and $1 \mathrm{mg} /$ $\mathrm{kg}$, respectively. $\mathrm{N}^{\mathrm{G}}$-Nitro-L-arginine methyl ester $\mathrm{L}-$ NAME, a NOS inhibitor) was subcutaneously injected 

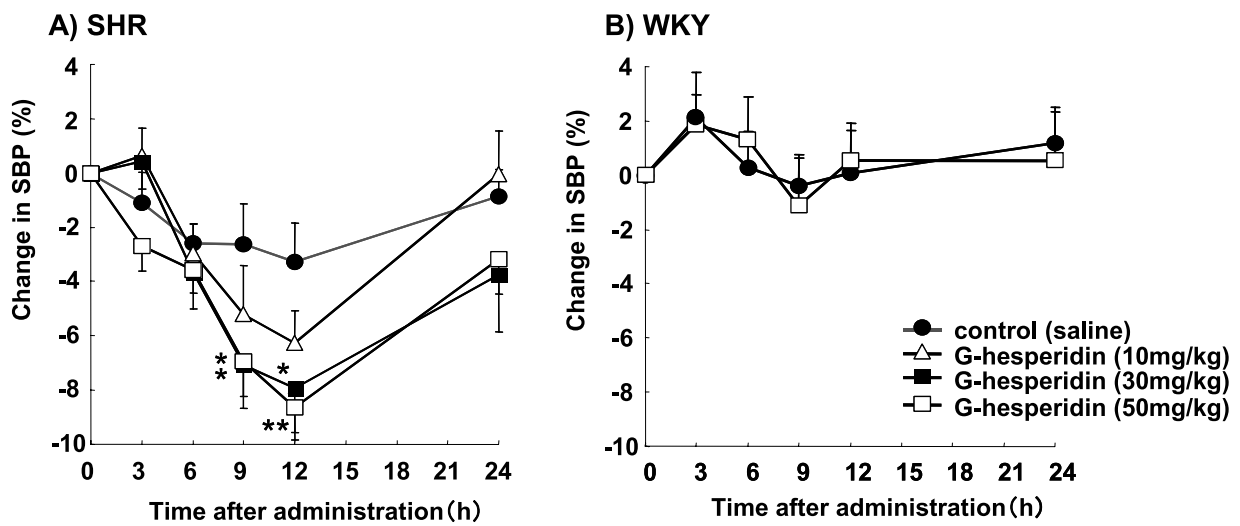

Fig. 1. Effects of single-oral administration of G-hesperidin on systolic blood pressure in SHR (A) and WKY (B). Change in SBP (\%) is expressed as the difference in SBP before and after administration. SBP, systolic blood pressure. Data are expressed as the mean \pm SE $(n=10-12) .{ }^{*} p<0.05,{ }^{* *} p<0.01$ vs. control group.

$(3 \mathrm{mg} / \mathrm{kg})$ into the neck $1 \mathrm{~h}$ before hesperetin administration. The control group received an intraperitoneal injection of $0.5 \%$ methylcellulose $(n=6-8)$.

Vascular reactivity studies. The vascular reactivity experiments were performed as described previously (11). The thoracic aorta rings from SHR or WKY were incubated with or without hesperetin at $10^{-5} \mathrm{M}$ for 20 min and then the vascular reactivity was measured after contraction by submaximal concentrations of phenylephrine $\left(10^{-6} \mathrm{M}\right)$. Endothelium-dependent and -independent relaxations were evaluated by concentrationresponse curves to acetylcholine (ACh, $10^{-9}$ to $10^{-5} \mathrm{M}$ ) and to sodium nitroprusside (SNP, $10^{-10}$ to $10^{-5} \mathrm{M}$ ), respectively. Relaxation was calculated as the percentage of precontractile vascular tone.

Statistical analysis. All data are presented as the means \pm SE. The significant differences were analyzed by the Dunnett test (Fig. 1) or Tukey-Kramer test (Table 1) for a post-hoc analysis using StatView ${ }^{\mathrm{TM}}$ (SAS Institute, Cary, North Carolina, USA). Comparisons of doseresponse curves were evaluated by 2-way ANOVA for repeated measures.

\section{Results and Discussion}

Changes in SBP after oral administration of G-hesperidin in SHR are shown in Fig. 1A. The initial SBP values were $204.8 \pm 2.1 \mathrm{mmHg}$ in the control group, and $210.3 \pm 2.5,201.8 \pm 2.3$ and $208.8 \pm 1.9 \mathrm{mmHg}$ in the 10,30 , and $50 \mathrm{mg} / \mathrm{kg}$ G-hesperidin groups, respectively. There were no significant changes in SBP in the control group throughout the experimental period. Ghesperidin decreased SBP in a dose-dependent manner; the changes in SBP $12 \mathrm{~h}$ after administration were $-6.2 \pm 1.2,-7.9 \pm 1.6$, and $-8.6 \pm 1.2 \%$ in the 10,30 , and $50 \mathrm{mg} / \mathrm{kg}$ G-hesperidin groups, respectively. In WKY, the initial SBP values were $130.1 \pm 1.4 \mathrm{mmHg}$ in the control group and $131.1 \pm 0.7 \mathrm{mmHg}$ in the $50 \mathrm{mg} /$ kg G-hesperidin group. G-hesperidin did not affect SBP in WKY throughout the experimental period (Fig. 1B). Heart rates were not affected by G-hesperidin administration throughout the experimental period in either SHR or WKY (data not shown).
Table 1. Combined effects of hesperetin and drugs on blood pressure in SHR.

\begin{tabular}{rlcc}
\hline Group & Drugs & $\begin{array}{c}\text { Hesperetin } \\
(50 \mathrm{mg} / \mathrm{kg})\end{array}$ & $\begin{array}{c}\text { Change in SBP } \\
(\%)\end{array}$ \\
\hline 1 & None & - & $0.8 \pm 1.1$ \\
2 & & + & $-12.3 \pm 1.2^{* *}$ \\
3 & Enalapril & - & $-12.5 \pm 2.5^{* *}$ \\
4 & & + & $-21.5 \pm 3.3^{* *, *}$ \\
5 & Nicardipine & - & $-10.9 \pm 1.3^{* *}$ \\
6 & & + & $-18.6 \pm 2.4^{* *, \dagger}$ \\
7 & Prazosin & - & $-26.2 \pm 1.9^{* *}$ \\
8 & & + & $-33.3 \pm 1.5^{* *, \dagger \dagger}$ \\
9 & L-NAME & - & $-1.3 \pm 0.9$ \\
10 & & + & $-5.8 \pm 1.5^{* *,+, \# \#}$
\end{tabular}

Change in SBP (\%) is expressed as the difference in SBP before and after administration of hesperetin and drugs. Data are expressed as the mean \pm SE $(n=6-8)$. SBP, systolic blood pressure.

${ }^{* *} p<0.01$ vs. group $1,{ }^{*} p<0.05$ vs. group $3,{ }^{\dagger} p<0.05$ vs. group $5,{ }^{\dagger \dagger} p<0.05$ vs. group $7,{ }^{\ddagger} p<0.05$ vs. group 9 , $\#$ \# $p<0.01$ vs. group 2.

In a previous study, continuous ingestion of hesperidin at a higher dose $(2 \mathrm{wk}, 200 \mathrm{mg} / \mathrm{kg})$ showed a diuresis effect in SHR (4). The urine volumes collected during the $12 \mathrm{~h}$ after G-hesperidin administration were $7.36 \pm 0.54$ and $7.73 \pm 0.54 \mathrm{~mL}$ in the control and Ghesperidin group, respectively. The urinary sodium concentrations were $83.5 \pm 8.2$ and $81.6 \pm 7.0 \mathrm{mEq} / \mathrm{L}$ in the control and G-hesperidin group, respectively. These data indicate that short-term administration of G-hesperidin did not affect diuresis in SHR.

It was assumed that orally ingested G-hesperidin undergoes the same metabolic process as hesperidin. Hesperidin is hydrolyzed to hesperetin and absorbed in the small intestine. In the present study, a significant hypotensive effect was observed 9 and $12 \mathrm{~h}$ after administration of G-hesperidin. The major metabolite of G-hesperidin, a glucuronide conjugate of hesperetin, peaks in serum $6 \mathrm{~h}$ after administration and remains 
A) SHR

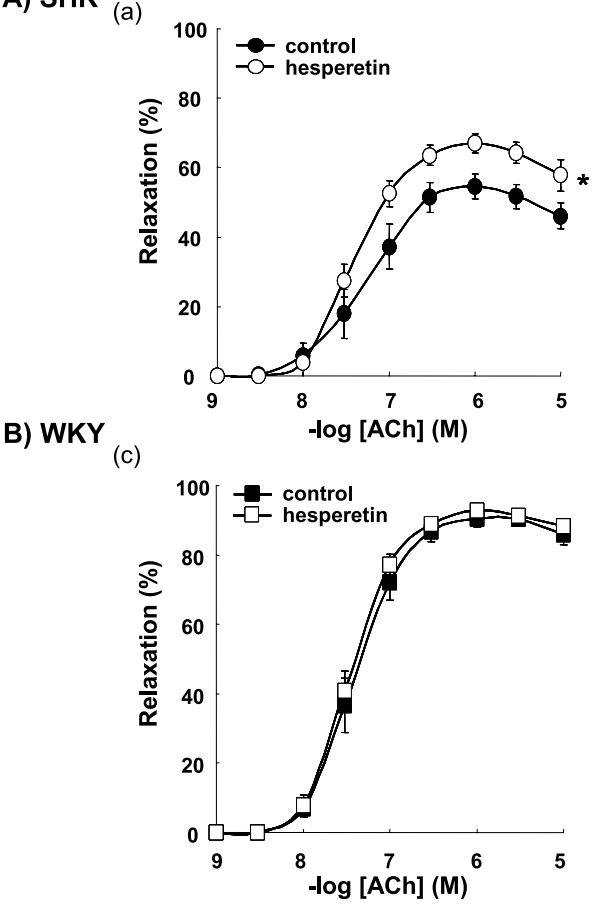

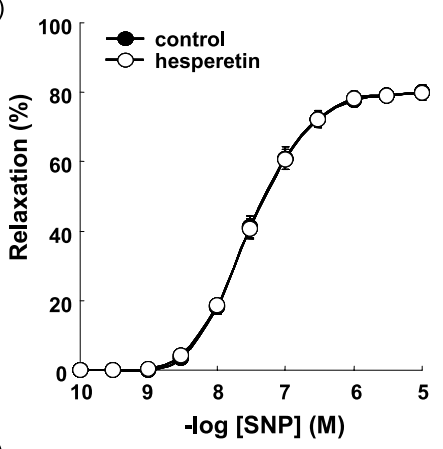

(d)

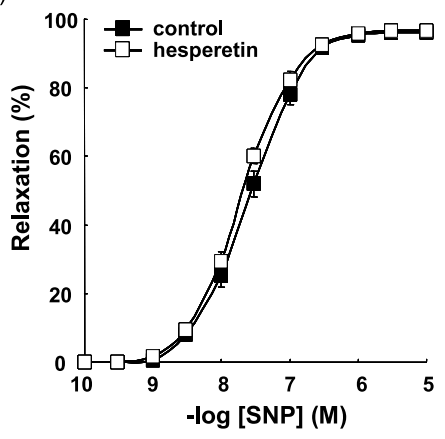

Fig. 2. Effects of hesperetin on endothelium-dependent relaxations induced by ACh (a and c) and endothelium-independent relaxations induced by SNP (b and d) in aortic rings from SHR (A) and WKY (B). Data are expressed as the mean \pm SE $(n=6-8) .{ }^{*} p<0.05$ vs. control group.

elevated until $12 \mathrm{~h}$ after administration (7). This result suggests that hesperetin metabolites may be responsible for the hypotensive effects of G-hesperidin. Therefore, the hypotensive responses to hesperetin alone and in combination with various inhibitors or blockers that affect blood pressure were examined (Table 1). In WKY, intraperitoneal injection of hesperetin did not affect SBP (data not shown). In SHR, hesperetin showed a significant hypotensive effect (decreased by $-12.3 \%$ ). When either enalapril, nicardipine, or prazosin was administered alone, SBP decreased significantly. The reduction in blood pressure was enhanced when these drugs were combined with hesperetin. In contrast, there was no change in SBP after subcutaneous injection of L-NAME alone. The hypotensive effect of hesperetin in the presence of L-NAME was significantly inhibited (decreased by $-5.8 \%$ ) compared to that of hesperetin alone. This result suggests that the hypotensive effect of hesperetin might be mediated by, at least in part, the vascular NOS pathway.

To test this hypothesis, the effects of hesperetin $\left(10^{-5} \mathrm{M}\right)$ on ACh-induced endothelium-dependent relaxation and SNP-induced endothelium-independent relaxation were investigated in isolated SHR and WKY aortas. In SHR aortas, hesperetin produced a significant increase in ACh-induced endothelium-dependent relaxation, but had no effect on endothelium-independent relaxation induced by SNP (Fig. 2A). In rat conduit arteries like the aorta, endothelium-dependent vasodilation in response to ACh was almost completely inhibited by L-NAME (NOS inhibitor), indicating that the reaction relies almost entirely on the endothelial release of NO (12). Endothelium-independent vasodilation induced by SNP was unaffected by hesperetin, indicating that increase in endothelium-dependent relaxation following hesperetin treatment is due to changes in endothelium-derived NO bioavailability. In contrast, in WKY aortas, no significant effects of hesperetin were observed in endothelium-dependent or -independent relaxation (Fig. 2B). Many investigators have recently reported that SHR arteries have increased superoxide anion production and inactivation of NO in vascular endothelial cells compared to WKY $(13,14)$. Hesperetin has been reported to show antioxidant activity $(15,16)$. This effect might contribute to improved NO bioavailability in endothelial cells in SHR. Several ex vivo studies have reported that hesperetin directly dilated the aorta in an endothelium-independent manner $(17,18)$. Our preliminary study confirmed endothelium-independent relaxation in response to hesperetin at high concentration (ex. $10^{-4} \mathrm{M}$ ). In the present study, we tested hesperetin at $10^{-5} \mathrm{M}$, which is close to the concentration observed after ingestion of G-hesperidin (7), allowing for examination of the effect of hesperetin on endothelial cells.

In summary, the hypotensive effect of hesperetin is associated with enhanced NO-mediated vasodilation. Therefore, the results of the present study provide novel evidence to identify the mechanisms by which G-hesperidin lowers blood pressure in hypertension.

\section{REFERENCES}

1) Bazzano LA, He J, Ogden LG, Loria CM, Vupputuri S, Myers L, Whelton PK. 2002. Fruit and vegetable intake and risk of cardiovascular disease in US adults: the first National Health and Nutrition Examination Survey Epi- 
demiologic Follow-up Study. Am J Clin Nutr 76: 93-99.

2) Garg A, Garg S, Zaneveld LJ, Singla AK. 2001. Chemistry and pharmacology of the citrus bioflavonoid hesperidin. Phytother Res 15: 655-669.

3) Wilmsen PK, Spada DS, Salvador M. 2005. Antioxidant activity of the flavonoid hesperidin in chemical and biological systems. J Agric Food Chem 53: 4757-4761.

4) Galati EM, Trovato A, Kirjavainen S, Forestieri AM, Rossitto A, Monforte MT. 1996. Biological effects of hesperidin, a citrus flavonoid. (Note III): antihypertensive and diuretic activity in rat. Il Farmaco 51: 219-221.

5) Bok SH, Lee SH, Park YB, Bae KH, Son KH, Jeong TS, Choi MS. 1999. Plasma and hepatic cholesterol and hepatic activities of 3-hydroxy-3-methyl-glutaryl-CoA reductase and acyl CoA: cholesterol transferase are lower in rats fed citrus peel extract or a mixture of citrus bioflavonoids. J Nutr 129: 1182-1185.

6) Hijiya H, Miyake T. 1991. Alpha-glycosyl hesperidin, and its preparation and uses. European Patent Publication No. 0402049.

7) Yamada M, Tanabe F, Arai N, Mitsuzumi H, Miwa Y, Kubota M, Chaen H, Kibata M. 2006. Bioavailability of glucosyl hesperidin in rats. Biosci Biotechnol Biochem 70: 1386-1394.

8) Ohtsuki K, Abe A, Mitsuzuwi H, Kondo M, Uemura K, Iwasaki Y, Kondo Y. 2002. Effects of long-term administration of hesperidin and glucosyl hesperidin to spontaneously hypertensive rats. J Nutr Sci Vitaminol 48: 420422.

9) Ohtsuki K, Abe A, Mitsuzumi H, Kondo M, Uemura K, Iwasaki Y, Kondo Y. 2003. Glucosyl hesperidin improves serum cholesterol composition and inhibits hypertrophy in vasculature. J Nutr Sci Vitaminol 49: 447-450.

10) Miwa Y, Mitsuzumi H, Sunayama T, Yamada M, Okada K, Kubota M, Chaen H, Mishima Y, Kibata M. 2005. Glucosyl hesperidin lowers serum triglyceride level in hypertriglyceridemic subjects through the improvement of very low-density lipoprotein metabolic abnormality. J Nutr Sci Vitaminol 51: 460-470.

11) Ulker S, McKeown PP, Bayraktutan U. 2003. Vitamins reverse endothelial dysfunction through regulation of eNOS and NAD $(\mathrm{P}) \mathrm{H}$ oxidase activities. Hypertension $\mathbf{4 1}$ : 534-539.

12) Nagao T, Illiano S, Vanhoutte PM. 1992. Heterogeneous distribution of endothelium-dependent relaxations resistant to $N^{\mathrm{G}}$-nitro-L-arginine in rats. Am J Physiol Heat Circ Physiol 263: H1090-H1094.

13) Zalba G, Beaumont FJ, San José G, Fortuño A, Fortuño MA, Etayo JC, Díez J. 2000. Vascular NADH/NADPH oxidase is involved in enhanced superoxide production in spontaneously hypertensive rats. Hypertension 35: 1055-1061.

14) Suzuki H, Swei A, Zweifach BW, Schmid-Schönbein GW. 1995. In vivo evidence for microvascular oxidative stress in spontaneously hypertensive rats: Hydroethidine microfluorography. Hypertension 25: 1083-1089.

15) Kim JY, Jung KJ, Choi JS, Chung HY. 2004. Hesperetin: a potent antioxidant against peroxynitrite. Free Radic Res 38: 761-769.

16) Hirata A, Murakami Y, Shoji M, Kadoma Y, Fujisawa S. 2005. Kinetics of radical-scavenging activity of hesperetin and hesperidin and their inhibitory activity on COX2 expression. Anticancer Res 25: 3367-3374.

17) Orallo F, Alvarez E, Basaran H, Lugnier C. 2004. Comparative study of the vasorelaxant activity, superoxidescavenging ability and cyclic nucleotide phosphodiesterase-inhibitory effects of hesperetin and hesperidin. Naunyn Schmiedebergs Arch Pharmacol 370: 452-463.

18) Calderone V, Chericoni S, Martinelli C, Testai L, Nardi A, Morelli I, Breschi MC, Martinotti E. 2004. Vasorelaxing effects of flavonoids: investigation on the possible involvement of potassium channels. Naunyn Schmiedebergs Arch Pharmacol 370: 290-298. 